\title{
A98-37099
}

AIAA-98-4294

\section{STUDY OF IN-FLIGHT REPLANNING DECISION AIDS}

\author{
Terence P. Fan*, Deborah S. Hyams*, and James K. Kuchar ${ }^{\dagger}$ \\ Department of Aeronautics and Astronautics \\ Massachusetts Institute of Technology \\ Cambridge, MA 02139
}

\begin{abstract}
A four-stage conceptual model of the in-flight replanning decision process is presented. The four stages, termed Monitor, Assess, Formulate, and Modify, are discussed along with their interrelationships. Information elements used in each stage are defined and grouped into three modes: supplemental, thresholded, and guidance. Each mode describes the manner in which automation processes and presents the information to the pilot. Additionally, results from a survey of pilot preferences and decisionmaking behavior are summarized. From the survey, weather information is cited as the most common element consulted during replanning. A case study from the survey is also described in which the effect of pilot reports of turbulence on pilot decision-making is examined.
\end{abstract}

\section{Introduction}

Air carrier flight plans are generally developed and modified according to the three different timescales shown in Fig. 1. Strategic plans are the result of a careful balance between flight schedule, environment (winds, weather, and traffic congestion), and aircraft performance, and they are designed to optimize the route so as to deliver the aircraft on time and with minimum fuel burn. Because of the complexity of the planning task, airline flight plans are developed hours before departure using dedicated personnel at an Airline Operations Center (AOC). The AOC has access to current weather, air traffic, and airline-specific information (e.g., schedules for aircraft, crew, and maintenance).

Along the flight path, localized, unexpected situations may occur, such as storm cells, traffic conflicts, or turbulence. These events may require a more tactical replanning effort. Tactical replanning takes place on the order of minutes, and generally involves temporary

\footnotetext{
* Graduate Research Assistant, Student Member AIAA

${ }^{\dagger}$ Assistant Professor, Member AIAA
}

route modifications designed to maintain safety and efficiency. Time-critical events (e.g., warnings from the Traffic Alert and Collision Avoidance System (TCAS) or from the Ground Proximity Warning System

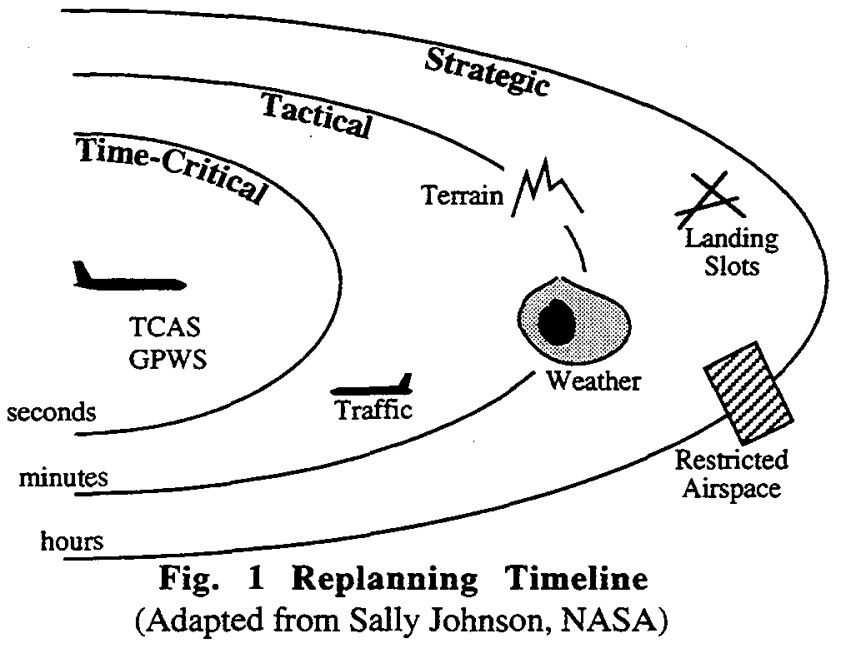

(GPWS)) require corrective action within a few seconds. The emphasis in time-critical events is on maintaining safety without regard for efficiency.

Depending on the timescale, the participants involved in replanning change. As described above, initial flight plans are generally developed at the AOC. While the aircraft is enroute, the AOC continues to play a major role in monitoring flight status and in making strategic replanning decisions in response to observed conditions that are significantly different from the forecast.

However, although the AOC has access to strategic weather information, it is the flight deck (either through on-board weather radar, pilot reports, or observed conditions) that has the most accurate and recent picture of the local environment. Accordingly, when short-term course or altitude changes are required (e.g., deviation around a storm cell), it is the pilots, combined with ATC, who currently make the bulk of the decisions. 
The AOC may not be directly involved in the tactical decision-making process because the pilots have access to more accurate information and also because there is insufficient time to confer with the AOC.

Additionally, increasing cockpit capabilities, such as sensors for the detection of clear air turbulence, enhanced ground proximity displays, and datalinked weather information, provide the foundation for improved flight-deck-based tactical decision-making. At the same time, these capabilities also raise questions with regards to the kinds of decisions that are appropriate for pilots to make, what information they would require, how this information can be managed most effectively, and how collaborative decision making between pilot, ATC, and AOC should be carried out.

To begin to address these issues, this paper presents a general model of the in-flight tactical replanning process as seen from the flight deck perspective. In addition, a survey of pilot preferences and decision-making behavior is summarized. To provide a manageable scope for discussion, this paper focuses on tactical in-flight replanning (for example due to convective weather or turbulence) performed on a glass-cockpit flight deck. Collaborative decision making issues between pilot, AOC, and ATC are certainly of critical importance, but are outside the scope of this initial study. Instead, this paper is targeted at the human-factors issues related to advanced decision aids in the cockpit.

\section{Prior Research}

Some in-flight replanning issues have been raised and explored in previous studies. ${ }^{1-10}$ Several of these studies focused on eliciting pilot behavior and preferences for replanning ${ }^{1-3}$ and on developing prototype expert systems to act as decision aids in the cockpit or for traffic management. ${ }^{4-7}$ Other studies have focused on modeling the replanning process and on outlining the major research issues. ${ }^{7-9}$ Additionally, NASA has undertaken an initiative to research and evaluate technologies for Advanced Weather Information (AWIN) for aircraft cockpits and has made recommendations for research in this area. ${ }^{10}$

Most recently, a research effort investigated the replanning process from the different perspectives of airlines, corporate operators, General Aviation aircraft, and ATC. ${ }^{9}$ This effort identified specific deficiencies in the current replanning process and recommended improving collaborative decision making between flight deck, AOC, and ATC. The study also advocated more advanced decision and collaboration aids in the cockpit to assist in the replanning process.

\section{Model of the Replanning Process}

Figure 2 shows a conceptual diagram of the general inflight replanning process. This model was adapted and modified from References 8 and 9 in order to more explicitly depict the interactions between stages in the replanning process. As shown, there are four main components of this model that represent basic processes in replanning: Monitor, Assess, Formulate, and Modify. Although the focus here is on the cockpit, a similar model can represent the replanning processes in the AOC and ATC.

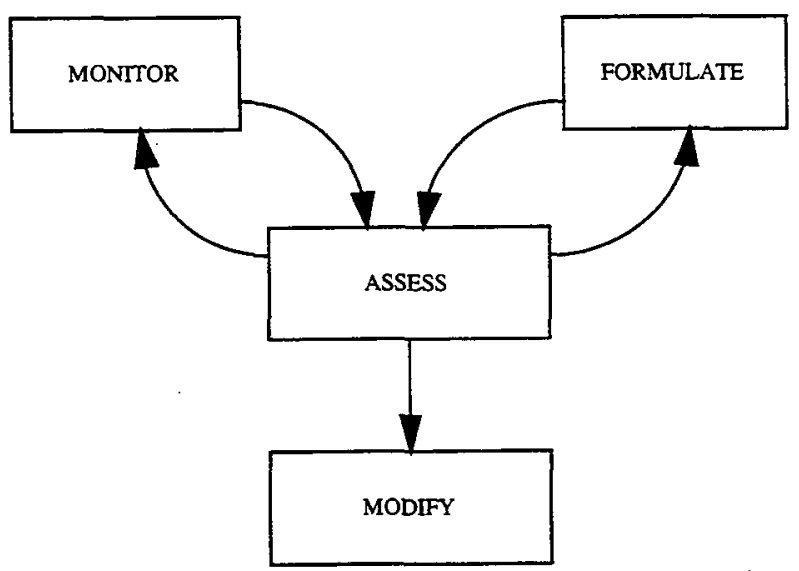

Fig. 2

Model of Replanning Decision Process

The first component in the model represents monitoring the current flight plan and the environment. Monitoring is the collection of information in order to determine the adequacy of the current flight plan. This step involves gathering relevant information from cockpit instruments, pilot reports, AOC, ATC, and observation out the windscreen. While monitoring, the pilot is watching his or her current flight path for problems or hazards.

Once the appropriate information is available, the next step is to assess the value of the plan that is being monitored. Assessment encompasses integrating the various sources of information and determining whether a plan is adequate. Thus, while monitoring is generally a data-collection task, assessment is a higher-level process requiring a value system and judgment.

The decision as to whether a flight plan is adequate can be modeled as a comparison against some form of threshold of acceptability. This threshold includes factors such as regulations, safety, efficiency, airplane performance constraints, as well as subjective preferences. In practice, due to the large number of variables involved, it is difficult to determine an explicit 
description of this process for use in an expert system or decision aid.

If assessment of the situation indicates that the current flight plan is adequate, then the monitoring and assessment cycle is continued. If, however, assessment indicates that the plan is inadequate (e.g., due to severe weather along the route of flight), the pilot will begin to formulate alternative plans. This task is similar to monitoring in that it primarily involves the collection of information needed to generate and evaluate deviations from the current flight plan. However, it differs from monitoring in that formulation of alternate routes is an active gathering of information in order to devise a new plan. This might include, for example, requesting ride reports or adjusting the weather radar elevation angle to examine precipitation returns at alternate altitudes. Potential changes in the flight plan must then be assessed in a manner similar to that discussed earlier. This assessment may result in the alternate plan being rejected, in which case further formulation and assessment cycling will be required.

Note also that the formulation task is not necessarily initiated by recognizing a deficiency in the current plan. Pilots generally formulate and assess alternate flight plans as a matter of course during a flight, both to determine whether a more preferable route is possible and also to 'stay ahead of the aircraft' should replanning be required at a later time.

The final step in replanning is the modify step. Modifying, in this model, represents physically implementing the new plan (e.g., by conferring with ATC and by programming the Flight Management System). Depending on the flight conditions, the modification may involve negotiations with and consent of ATC and/or the AOC. Thus, should a proposed route change not be acceptable to ATC or AOC, additional iterations of assessment and formulation may be required. This hierarchy of decision-making is one area in which the current replanning process is inefficient: pilots may develop a tactical change in flight plan that subsequently is rejected by ATC or AOC, necessitating additional replanning. If ATC, AOC, and the flight crew are all more directly involved in decision-making early on, the frequency of such iteration may be reduced.

\section{Information Processing}

The monitoring, assessment, and formulation processes depend on a number of information elements upon which decisions are based. These information elements can be grouped into four categories: hazard, efficiency, comfort, and constraint. Hazard information includes external factors that may pose a threat to safety, including terrain, traffic, severe weather, icing, or windshear. Efficiency considerations include winds, fuel burn, and flight time. Comfort considerations include ride quality, turbulence, and workload in developing, implementing, and monitoring the flight plan. Finally, constraints include aircraft performance, airspace restrictions, information accuracy, and system failures or degraded operation.

Any flight replanning decision requires a balance between these four information categories. For example, maintaining a large margin around weather improves safety and ride quality, but negatively impacts efficiency. Orchestrating this balance requires knowledge at some level of the degree of importance of each element. Automation can provide this knowledge through one or more modes of information processing, discussed below.

Each of these information elements may be processed and displayed in one of three modes: supplemental, thresholded, or guidance. In a supplemental mode, the information is passively presented without any automated judgment or bias. Examples of supplemental information are traffic icons, precipitation returns from radar, or forecast winds. This information is then used by the decision maker, who integrates it with other considerations to determine the best course of action.

In a thresholded mode, automation determines that some parameter requires attention and advises the decision maker. Thresholded information could include a Traffic Advisory or feedback that a flight plan will not clear terrain or satisfy fuel constraints. Thus, with thresholded information, the automation has been designed with preset definitions of acceptable and unacceptable states. It is then important to ensure that the automation's distinction between acceptable and unacceptable is a reasonable match with the pilot's point of view. If the pilot and automation disagree, trust in the decision aid may drop and the automation may not be used. ${ }^{11}$

Finally, guidance information provides the decision maker with a suggested or commanded course of action. Guidance information could include flight director bars or computer-generated optimal flight plans, and thus typically requires a higher degree of processing logic than the other two modes. Again, the automated guidance must be in close conformity with strategies that the pilot would use or else the automation will not be used or trusted.

Although moving from supplemental to thresholded to guidance information requires an increase in automation sophistication, it is not correct to say that the lower 
modes are not important. Providing guidance or thresholded information without corroborating supplemental information may lead to pilot distrust of the automation. Such effects have been observed, for example, in early versions of the Ground Proximity Warning System (GPWS), which produced terrain alerts without any supplemental information with which to validate the alert. ${ }^{12}$ Recent Enhanced GPWS displays now also provide a view of the terrain so that the pilot has the supplemental information with which to verify and trust the automation.

To be effective, thresholded and guidance information require integrating a number of elements together. For example, rerouting suggestions cannot be consistently formulated without access to weather, traffic, and restricted airspace information. Due to the complexity of doing so, in-flight replanning information is currently presented only in supplemental form. It is the pilot's task to then perform integration between the information elements. However, due to the workload involved, it may be appropriate in some cases to provide automated thresholded or guidance-based information that could advise the pilot when evaluating alternate routes.

The appropriate level of automated integration depends to a large degree on the type of information that is being presented and its role in the decision process. For example, incomplete supplemental information may be best left unintegrated. This allows the pilot to integrate the information using knowledge of the environment that is not available to automation. Also, an automated system that integrates faulty information may be less acceptable than one which does not attempt such advanced processing. However, guidance information may be required to be fully integrated, as it is undesirable to produce automated suggestions that are unacceptable because of constraints not considered by the automation. For example, automated traffic rerouting guidance that repeatedly suggests flight plans into severe weather may reduce pilot confidence in automation, lead to confusion, and ultimately pilot deactivation of the automation.

\section{In-Flight Replanning Information Survey}

To better understand how information is used in the replanning process on the flight deck, a survey of active pilots was conducted. To allow for efficient distribution to pilots around the world, the survey was conducted via the world wide web. Users could fill out the survey using a web browser, and their responses were delivered via anonymous email to the experimenters.
A hypertext link to the survey was posted at several popular aviation websites, including a general-interest web site that produces weekly newsletters to aviation enthusiasts (Avweb ${ }^{13}$ ) and a site devoted to users of Flight Management Systems (Bluecoat ${ }^{14}$ ). Messages about the survey were also sent to a number of domestic airlines, but feedback was not received as to whether this information was distributed to their pilots. Because of the inability to control and verify the background of respondents, several open-ended questions on the survey were used to screen out non-pilots.

Over a three-week period, from mid-January to early February, 1998, a total of 309 valid survey responses were received. These surveys came from a variety of pilots with different levels of flight experience. The respondents were grouped into four categories according to the highest level of flight rating obtained: Private, Instrument, Commercial, and Air Transport Pilot. Among the 309 respondents, 91 (29\%) were Air Transport Pilots. Because the focus of this effort was on airline in-flight replanning, the data discussed in this paper include only the responses from the Air Transport Pilots. A complete summary of the survey responses for all pilot types is available in Reference 15.

Among the Air Transport Pilots, 30\% flew primarily within the U.S., 30\% within North America outside of the U.S., and $40 \%$ flew primarily international routes. In particular, there were at least 5 airline pilots with home bases outside of North America. A total of $47 \%$ of the pilots indicated that they flew primarily for scheduled air carriers, $24 \%$ for corporate businesses, and $9 \%$ each for chartered carriers, flight instruction, and pleasure. The pilots had an average of 9,678 total flight hours with an average age of 44 years.

The results of the survey are briefly discussed below, and they are organized according to the model of the inflight replanning process introduced above.

\section{Monitoring}

Since the monitoring stage is used to collect data in order to determine the acceptability of the current flight plan, it is important to know what situations initiate replanning. In the survey, pilots were asked to identify the condition(s) that usually led them to initiate the inflight replanning process.

Fig. 3 shows the conditions that were cited by at least 5 respondents (via a free response question) as initiators of the replanning process. Weather was by far the most commonly cited cause, followed by fuel, traffic delay at destination, and wind conditions. 
The free-response format was used to force the pilots to think from their own experience, but one drawback of this format is that the terminology used to describe the same conditions may vary. For instance, some pilots listed "diversion to alternate [airport]" as a cause for them to replan. However, these cases might be consequences of weather, mechanical problems, or lower-than-expected fuel levels, each of which were cited specifically by other subjects.

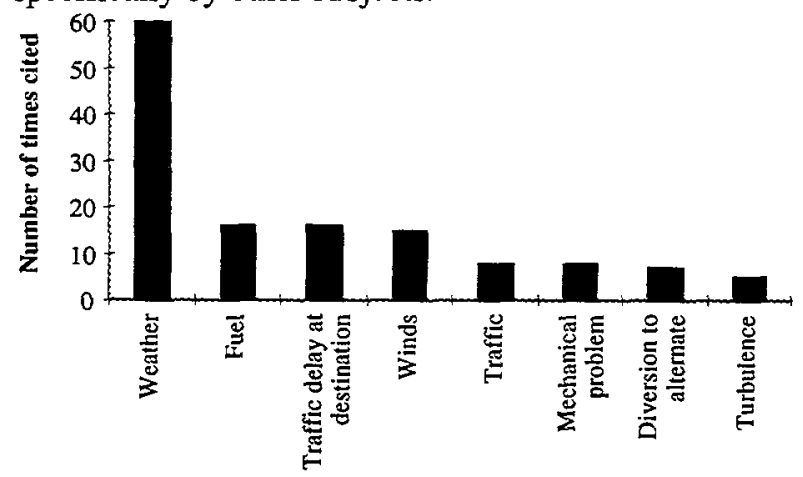

Fig. 3 Initiators of Flight Replanning (Cited 5 times or more)

\section{Formulating Alternate Flight Plans}

In a free-response question, pilots were asked to list the information sources they consult when forming alternate flight plans. These sources included information gathered both pre-flight and in-flight. Fig. 4 shows the number of times each different source was quoted by subjects in their response to this question.

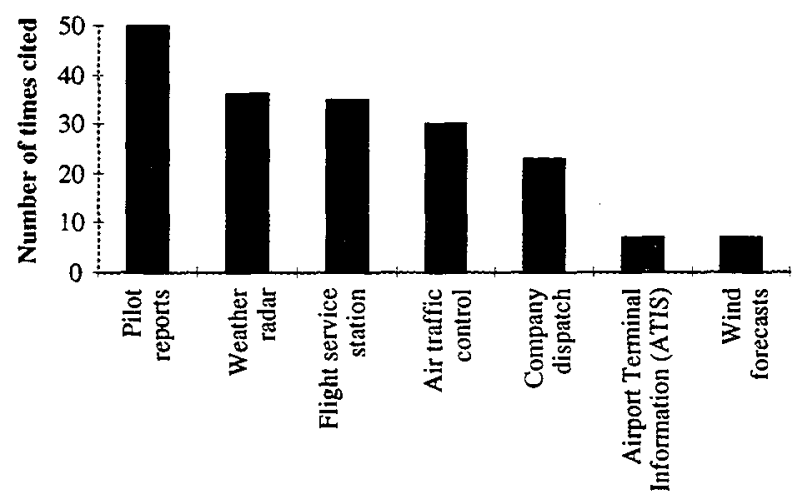

Fig. 4 Replanning Information Sources (Cited 5 times or more)

Pilot Reports are by far the most commonly reported source, followed by information from weather radar, flight service stations, air traffic controllers, company dispatchers, airport terminal information service (ATIS), and wind forecasts. Predictably, the most commonly cited sources are the ones which provide the only realtime local sources of weather information to the pilot other than the view out of the windscreen.
Pilots were also asked, in a free-response question, to describe what conditions would lead to a change in altitude, heading, or speed when they were replanning. The most common conditions (cited more than 10 times) leading to heading changes were deviations around weather systems. Altitude changes were most commonly induced by unfavorable winds, icing, or turbulence. Speed changes were most often initiated in response to turbulence or schedule adherence.

\section{Assessment}

After the pilot collects information, he or she may place values on the information based on perceived levels of importance of the varying elements. To determine these levels, another survey question dealt with the importance that pilots place on several specified types of information in their replanning decisions. These elements include: wind (aloft) information, nearby traffic, terrain, weather systems, turbulence, icing, fuel consumption, and restricted airspace. The pilots were asked to rate the level of importance on a scale from 1 (least important) to 10 (most important). The mean ratings are shown in Figure 5.

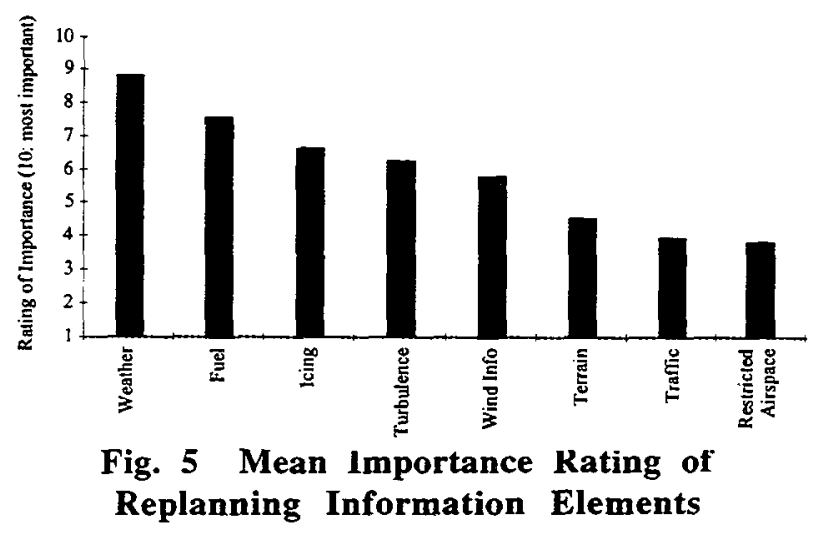

As expected, weather systems (precipitation) were rated as the most important factor affecting flight replanning decisions. Another observation from Figure 5 is the relatively low rating for traffic information and restricted airspace. This reflects the extent to which pilots have information and responsibility for these elements. Although traffic information from onboard Traffic Alert and Collision Avoidance Systems (TCAS) is available, this information is too uncertain to be of use in inflight replanning. ATC retains the responsibility for vectoring other traffic in response to a request for a change in flight plan. Additionally, ATC may deny or amend a routing request due to traffic. In some proposed free flight operating environments, however, some of this responsibility may fall on the flight crew. In order to maintain a similar level of mental workload for 
pilots under free flight (compared to the present), a wellintegrated decision support system is clearly needed.

After determining possible options for alternate flight plans, it is then the job of the pilot to select one plan for implementation. In order to determine methods that pilots use to choose which course is most suitable for implementation, several survey questions addressed the priority of decision attributes as well as the particular replanning actions which are taken in response to specific situations.

In order to understand how pilots might choose between several replanning scenarios it is important to understand the priority that pilots place on different decision attributes. To this end, pilots were asked to rank the following flight objectives in the order of importance, with 1 being the most important and 5 being the least: safety, ride comfort, fuel efficiency, schedule adherence, and workload. The average ranking of these attributes by air transport pilots is shown in Table 1 .

Table 1:

\begin{tabular}{cc}
$\begin{array}{c}\text { Mean Ranking of } \\
\text { (1=most important, } \\
\text { Category }\end{array}$ & $\begin{array}{c}\text { Decision Attributes } \\
\text { Sean Ranking (Std dev) }\end{array}$ \\
\hline \hline Safety & $1.07(0.45)$ \\
Ride Quality & $3.13(1.18)$ \\
Schedule Adherence & $3.47(1.11)$ \\
Workload & $3.64(1.21)$ \\
Fuel Efficiency & $3.69(\mathbf{0 . 9 6 )}$ \\
\hline \hline
\end{tabular}

As is evident from the table, safety is clearly a prime concern for flying, and it is rated higher than other options by a statistically significant margin $(p<0.01)$. After safety, ride quality and schedule adherence were ranked in order of increasing importance $(p<0.05)$. The differences in ranking between ride quality and workload and between ride quality and fuel efficiency are also statistically significant $(p<0.05)$. The proximity of rankings of the last three attributes points to the fact that one or more of these factors may be traded off for another, depending on the situation. The results in Table 1 are consistent with those obtained in a similar survey of long-haul pilots. ${ }^{1}$

The final step of the assessment process is determining whether a proposed alternate flight plan should be implemented. In the survey, pilots were asked to estimate their replanning acceptance rate, defined as the fraction of alternate plans that they formulate that the pilots deem are good enough to warrant implementing. Note that the acceptance rate as defined here does not include considerations of obtaining clearance from ATC or AOC; the rate discussed here is solely the pilot's internal acceptance rate of potential replan decisions.

The number of subjects that indicated a given acceptance rate were totaled, as summarized in Fig. 6. As shown, more than half of the respondents estimated that they have an acceptance rate of $20 \%$ or lower. Nearly all (90\%) of the respondents estimated their acceptance rate to be below $70 \%$. Thus, a relatively large fraction of replanning appears to be performed mentally without ever reaching the implementation stage.

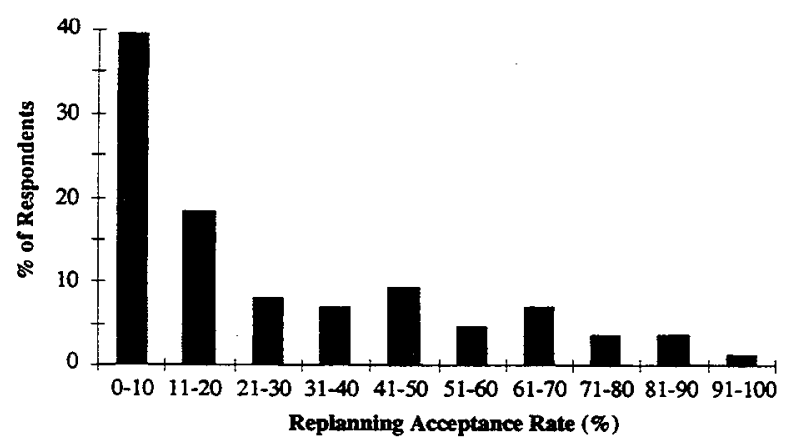

Fig. 6 Replanning Acceptance Rate

\section{Effect of Pilot Reports in Replanning}

In addition to the general information processing behavior described above, the survey incorporated a case study to explore the use of pilot reports when replanning. The pilots were presented with 5 separate scenarios involving the prospect of turbulent conditions (Table 2). In every scenario, pilots were told that there was a region approximately 20 minutes ahead along their route of flight in which moderate chop (turbulence) had been reported earlier. Moderate chop is a state of continuous, rapid turbulence, which may be dangerous to unbelted passengers, but is not dangerous for flight.

Scenario A was a control case, in which the subject's aircraft was the only aircraft in the area. Pilot decisionmaking was therefore based solely on the prior report of turbulence 20 minutes ahead.

In scenario $B$, the pilots heard from the radio that another airplane approximately 5 to 10 minutes ahead at the same flight altitude was proceeding along the same route, but made no turbulence report. This scenario was designed to determine whether a "Tead" aircraft could have an effect on pilot decision-making.

In scenario $C$, the pilots heard a report that the lead airplane was experiencing moderate chop. Comparing scenario $\mathrm{C}$ with scenarios $\mathrm{A}$ and $\mathrm{B}$ allows evaluation of the effect of a pilot report from a lead aircraft. 
In scenario $\mathrm{D}$, the lead aircraft, in addition to the pilot report of moderate chop, also requested a change in altitude in order to improve ride quality. In this case, the additional impact of a lead aircraft that changes its flight plan can be determined.

Finally, in scenario E, the lead airplane was proceeding straight and had not reported any turbulence, but the subject's airplane was experiencing moderate chop. This allows for the exploration of the effect of actual (as opposed to predicted) turbulence on decision-making.

Table 2: Turbulence Case Study Scenarios

\begin{tabular}{c|c|c} 
Scenario & Subject Aircraft & Lead Aircraft \\
\hline \hline A & no turbulence & - \\
\hline B & no turbulence & no report \\
\hline C & no turbulence & moderate chop \\
\hline D & no turbulence & $\begin{array}{c}\text { moderate chop and } \\
\text { altitude change request }\end{array}$ \\
\hline E & moderate chop & no report \\
\hline \hline
\end{tabular}

In each scenario, respondents were asked to choose one of three options: proceed using the current flight plan, change the flight plan, or request more information. In addition, there was space for pilots to specify the type of information they would request and to give general comments with regard to the scenario.

The results of the scenarios are reported below. Only results which are statistically significant at the $1 \%$ level are discussed $(p<0.01)$.

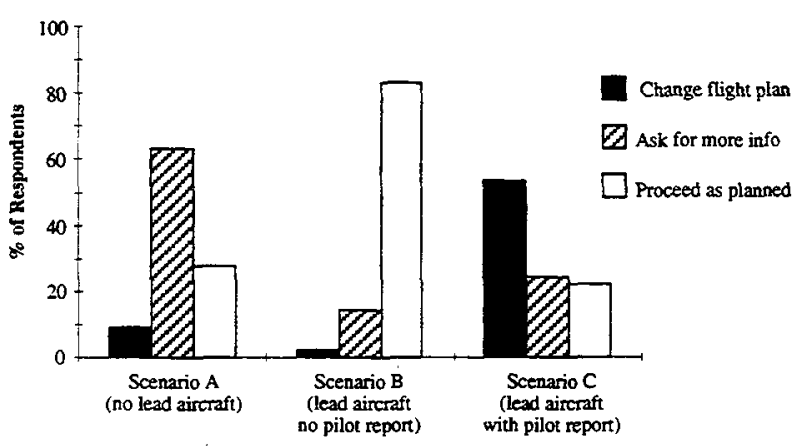

Fig. 7 Pilot Report Effects

(Scenarios A, B, and C)

In each of the first three scenarios $(A, B$, and $C)$, there were clearly-preferred courses of action that received a majority of support by the respondents (Fig. 7). Whether this preferred course of action was to continue with the current plan, replan, or to request more information depended on the specific scenario, as discussed below.
In scenario A, $63 \%$ of the pilots indicated that they would request more information before deciding whether to continue with the current flight plan. This likely results from the ambiguous baseline condition in which the recency and scope of the turbulence report was not explicitly mentioned to the subject.

In scenario $B$, pilot preference shifted significantly such that $83 \%$ indicated that they would continue on course. It therefore appears that the presence of a lead aircraft can have a significant influence on the pilots' replanning decisions to proceed as planned. This implies that the pilots are reassured by the presence of another aircraft, presumably because they expect the lead aircraft to report turbulence when and if any is encountered. Thus, the lead aircraft acts as a surrogate ride quality sensor.

Scenario $\mathrm{C}$ similarly resulted in a significant change in pilot preference. In this case, slightly more than half (53\%) indicated that they would request a change in flight plan. The remaining pilot preference was split approximately equally between requesting more information and proceeding as planned. This scenario shows that a pilot report of turbulence from a lead aircraft shifts pilot preference toward changing the flight plan. However, approximately $25 \%$ of the pilots indicated that they would request more information, suggesting that pilot reports, though useful, do not provide all of the information that is desired.

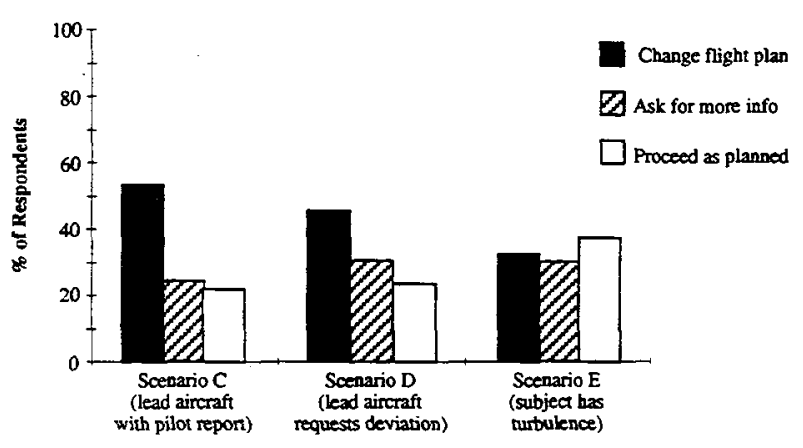

Fig. 8 Pilot Report Effects (Scenarios C, D, and E)

The fourth scenario, $D$, was designed to test the additional effect caused by a deviation request from a lead aircraft. In this scenario, the fraction of pilots requesting a change of plan was approximately equal to that in scenario $\mathrm{C}$, as shown in Fig. 8. Apparently, a request for deviation due to turbulence does not carry much additional information over a turbulence report alone, and therefore does not significantly change decision-making. 
Finally, in scenario E (also shown in Fig. 8), a somewhat surprising result was obtained. Fewer pilots $(33 \%)$ indicated that they would request a change in their flight plan in this case than had indicated they would do so in scenarios $C(53 \%)$ or D (45\%). That is, subjects were less willing to change the flight plan while they were experiencing turbulence than when the subject's ride was smooth. The reason for this apparent contradiction is likely due to the presence of the lead aircraft. Namely, a lead aircraft that is not reporting turbulence is interpreted to indicate that there is no turbulence at its location. Thus, in scenario $E$, the subjects likely decided that the turbulence would abate in the near future, and thus a change of altitude might not be warranted. In contrast, although the subject's ride was smooth in scenarios $C$ and $D$, the prospect of turbulence ahead, due to the pilot report, was sufficient to lead many subjects to request a change in flight plan.

In addition to selecting between the three options, several subjects wrote comments regarding what additional information they would have liked to have had in the scenarios. The types of additional information requested in scenario included: alternate altitudes or flight paths with smoother air (mentioned by 13 pilots); type of aircraft reporting the turbulence (by 10 pilots); spatial extent of the problem, or how long turbulent conditions would last (by 9 pilots); and the ride quality of additional aircraft in the area (by 7 pilots). Other factors that would also be considered by the air transport pilots (as expressed in the additional comments for this question) included cabin concerns (i.e., whether meals are being served, mentioned by 5 pilots), the recency of the pilot report (mentioned by 3 pilots), fuel (mentioned twice) and penalty of diversion (mentioned twice). In the general comments section, diversion penalties, weather conditions, wind profile, airborne traffic, and cabin concerns (mentioned once or twice each) were also listed as other factors influencing the replanning decisions.

Interestingly, in scenario $\mathrm{E}$, seven pilots requested a ride quality report from the lead airplane, and only four pilots mentioned that would report their choppy conditions to ATC.

\section{Conclusion}

The current technological environment is such that improved cockpit decision aids for in-flight replanning are becoming possible. As past experience has shown, however, any enhancements to the cockpit need to be carefully engineered so as to ensure an improvement in performance rather than a detriment or potential for a safety hazard. The ability to add features to the cockpit does not necessarily imply desirability.
This project has developed a preliminary model of inflight replanning and has explored current pilot decisionmaking behavior and preferences for flight replanning automation based on that model.

In the survey of pilots, weather concerns are cited as the most common initiators of replanning. The most commonly cited sources of information used during replanning are pilot reports and weather radar. These information sources correspond both to the most common causes of replanning (weather) and to the fact that they are essentially the only real-time local sources of weather information available to the pilot other than the view out the windscreen.

Pilot reports appear to play a significant role in decision-making related to turbulence. A pilot report of turbulence from a leading aircraft along the route of flight significantly alters the following-pilot's preference from "request more information" to "change flight plan". The lack of a pilot report from another aircraft in an area of previously-reported turbulence likewise significantly alters pilot preference from "request more information" to "proceed as planned". An aircraft that issues a pilot report for turbulence and also requests a change in altitude does not significantly alter following-pilot preferences to change course over that which occurs when the pilot report alone occurs, suggesting that the deviation request does not provide much additional information over the ride report itself.

Finally, it is important to note that any changes in the cockpit replanning environment need to also be evaluated in terms of their larger impact on collaborative decision making between pilot, air traffic controller, and airline. Potential disadvantages of enhancing cockpit decision aids are that there may be a greater likelihood of conflict in decisions between the parties involved, there may be increased pilot workload or distraction, and the appropriate roles of each of the players may be unclear or misunderstood. Thus further study on cockpit decision aids needs to be included as part of a larger effort that also examines impacts on $\mathrm{ATC}$ and $\mathrm{AOC}$

\section{Acknowledgments}

The authors are appreciative of the support provided by the NASA Langley Research Center, including Sally Johnson, Mark Ballin, and Terry Abbott. We are also appreciative of the collaboration with Bill Corwin at Honeywell, and Geoff Gosling at the University of California, Berkeley. The pilot respondents to the survey also deserve thanks for their time and input. 
This research was supported by the National Center of Excellence for Aviation Operations Research, under Federal Aviation Administration Research Grant Number 96-C-001. This document has not been reviewed by the Federal Aviation Administration (FAA). Any opinions expressed herein do not necessarily reflect those of the FAA or the U.S. Department of Transportation.

\section{References}

${ }^{1}$ Patrick, N. J. M., "Decision-Aiding and Optimization for Vertical Navigation of Long-Haul Aircraft", $\mathrm{PhD}$ Thesis, Massachusetts Institute of Technology, Cambridge, MA, August, 1996.

${ }^{2}$ Midkiff, A., and R. J. Hansman, "Identification of Important Party Line Informational Elements and the Implications for Situational Awareness in the Datalink Environment", MTT Aeronautical Systems Laboratory Report ASL-92-2, Massachusetts Institute of Technology, Cambridge, MA, May 7, 1992.

${ }^{3}$ Dershowitz, A., "The Effect of Options on Pilot Decision Making in the Presence of Risk", $\mathrm{PhD}$ Thesis, Massachusetts Institute of Technology, Cambridge, MA, October, 1997.

${ }^{4}$ Prevot, Gerlach, Ruckdeschel, Wittig, and Onken, "Evaluation of Intelligent On-Board Pilot Assistance in In-Flight Field Trials", 6th IFAC Symposium on Analysis, Design, and Evaluation of Man-Machine Systems, Cambridge, MA, June 27-29, 1995.

${ }^{5}$ Robinson, Davis, and Isaacson, "Fuzzy ReasoningBased Sequencing of Arrival Aircraft in the Terminal Area", AIAA Guidance Navigation and Control Conference, New Orleans, LA, August, 1997.

${ }^{6}$ Nguyen, T., and D. Ward, "A Neural-Network Based Inference Engine for a General Aviation Pilot Advisor", $35^{\text {th }}$ AIAA Aerospace Sciences Meeting and Exhibit, Reno, NV, January 6-10, 1997.

${ }^{7}$ Rudolph, Homoki, and Sexton, "Diverter: Decision Aiding for In-Flight Diversion", NASA CR$182070,1991$.

${ }^{8}$ Abbott, T., "Functional Categories for Future Flight Deck Designs", NASA-TM-109005, Hampton, VA, August, 1993.

${ }^{9}$ Rogers, Corwin, Riley, Quarry, and Dwyer, "Airborne-Based Conflict Probe", NASA Contractor Report, February, 1998.

${ }^{10}$ NASA Aviation Safety Investment Strategy Team (ASIST), "Weather Investment Recommendations Summary", NASA Langley Research Center, April $15,1997$.
${ }^{11}$ Pritchett, A., "Pilot Non-Conformance to Alerting System Commands During Closely Spaced Paralle Approaches", PhD Thesis, Massachusetts Institute of Technology, Cambridge, MA, January, 1997.

${ }^{12}$ DeCelles, J. L., "The Delayed GPWS Response Syndrome", Technical Report, Aviation Research \& Education Foundation, Herndon, VA. July, 1991.

13 www.avweb.com

${ }^{14}$ www.NeoSoft.com/ sky/BLUECOAT

15 Fan, T., Hyams, D., and J. Kuchar, "Preliminary Study of In-Flight Replanning Performed on the Flight Deck", National Center of Excellence in Aviation Operations Research, NEXTOR Report RR-98-4, MIT, Cambridge, MA, April 30, 1998. 